\title{
Endometrial stromal nodule: a rare case report
}

\section{Shreya Bhattarai, Taru Gupta, Jagriti Bhardwaj*, Aanchal Sablok}

Department of Obstetrics and Gynecology, ESI PGIMSR, Basaidarapur, New Delhi, India

Received: 21 February 2020

Revised: 21 March 2020

Accepted: 27 March 2020

\section{*Correspondence:}

Dr. Jagriti Bhardwaj,

E-mail: jagriti.bhardwaj@gmail.com

Copyright: (c) the author(s), publisher and licensee Medip Academy. This is an open-access article distributed under the terms of the Creative Commons Attribution Non-Commercial License, which permits unrestricted non-commercial use, distribution, and reproduction in any medium, provided the original work is properly cited.

\begin{abstract}
Endometrial stromal nodules (ESN) are benign tumours of mesenchymal origin with features reminiscent of proliferative phase endometrial stroma. Diagnosis of ESNs can be established only by light microscopy and no preoperative diagnostic methods are available. Although ESNs are benign and rare, distinguishing it from other types of invasive stromal tumours is of utmost importance since prognosis and management change considerably with the diagnosis. This was a rare case report of endometrial stromal nodule in a nulliparous woman, 30 years old who presented with complaint of menorrhagia and primary infertility and had a preoperative diagnosis of large leiomyoma with cystic degeneration. She underwent a fertility preserving conservative surgery i.e. myomectomy via abdominal route, histopathology reports of which revealed endometrial stromal nodule that changed the final diagnosis and follow up regime of the patient.
\end{abstract}

Keywords: Endometrial stromal nodules, Myomectomy, Nulliparous woman

\section{INTRODUCTION}

Endometrial stromal nodules are a rare subtype of endometrial stromal tumours (ESTs). ESTs account for $3 \%$ of all uterine neoplasms and are diagnosed in most instances by light microscopy. ${ }^{1} 1 / 4^{\text {th }}$ of these tumours are endometrial stromal nodule. They occur at any age ranging from 31 to 86 years with a mean of 54 years. $^{2}$

In latest World health organization classification (2014), ESTs are divided into ${ }^{3}$ :

- Endometrial stromal nodule (ESN) - least common

- Low grade endometrial stromal sarcoma (LG-ESS)

- High grade endometrial stromal sarcoma (HG-ESS)

- Undifferentiated endometrial sarcoma (USS).

Based on their histologic appearance, ESN and low grade ESS fall in the lower end of the spectrum of ESTs. ESN is cytologically similar to low grade sarcomas. Both are composed of a diffuse growth of uniform small blue cells with scant cytoplasm and oval to spindle nuclei that resemble endometrial stromal cells of proliferative endometrium, that grow in sheets and focally whorl around arterioles. ESN is differentiated from LG-ESS by its an expansile but non infiltrative and smooth margins in contrast with an irregular nodular growth in endometrium with varying degrees of myometrial permeation as well as extension to extrauterine veins and lymphatics seen in the latter. Microscopically, myometrial infiltration if present, can be seen as protrusions less than 3 in number of $3 \mathrm{~mm}$ or less with no angiolymphatic invasion and minimal mitotic activity $(<3 / 10 \mathrm{HPF}) 3$ in a stromal nodule. Tumours with myometrial infiltration $>3 \mathrm{~mm}$ and $>3$ in number would be categorized as LG-ESS.

High grade endometrial stromal sarcomas and undifferentiated endometrial stromal sarcomas show destructive infiltrative growth into the endometrium or myometrium or both. Microscopically, marked nuclear 
atypia is characteristic of high grade ESS and UES with mitotic rate $>10 / 10 \mathrm{HPF}$.

The non-infiltrative border of stromal tumours is the single most important criterion for the diagnosis. Histological examination of the uterus is the most accurate method to make an appropriate diagnosis, thus hysterectomy being the treatment of choice in benign as well as malignant tumours. In this study we describe a young nulliparous patient who underwent conservative fertility preserving surgery and resection of mass which was postoperatively diagnosed as endometrial stromal nodule.

\section{CASE REPORT}

Mrs X, 30 years old, nulliparous presented with heavy menstrual bleeding and spasmodic dysmenorrhea since 1 year associated with no other complains. On general examination patient was of average built, well nourished, she was clinically pale. Abdominopelvic examination showed uterus enlarged to 16 weeks size of gravid uterus, soft to firm in consistency, non-tender, mobile, smooth surfaces, and lower limit could not be reached. On per vaginal examination uterus was 16 weeks of size, mobile, non-tender, a large fibroid like mass was felt on posterior wall of uterus.

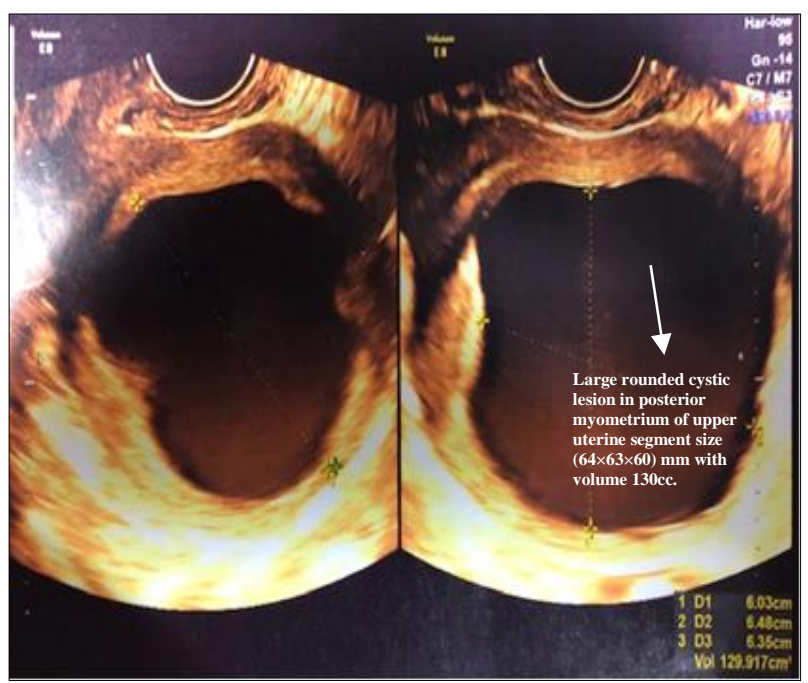

Figure 1: Transvaginal USG of a large posterior wall fibroid with cystic degeneration.

Transabdominal and transvaginal sonography (Figure 1) showed a well-defined, large rounded cystic lesion in posterior myometrium of uterus of size $64 \times 63 \times 60 \mathrm{~mm}$ with volume of $129 \mathrm{cc}$ suggestive of intramural fibroid with cystic degeneration, bilateral ovaries appeared normal. MRI showed diffuse and asymmetrical uterus with diffuse scattered hyperintense foci in posterior myometrium. Bilateral adnexa were grossly normal (Figure 2). After being transfused for anemia, a fertility preserving treatment was discussed with the patient and a myomectomy via abdominal route was performed.

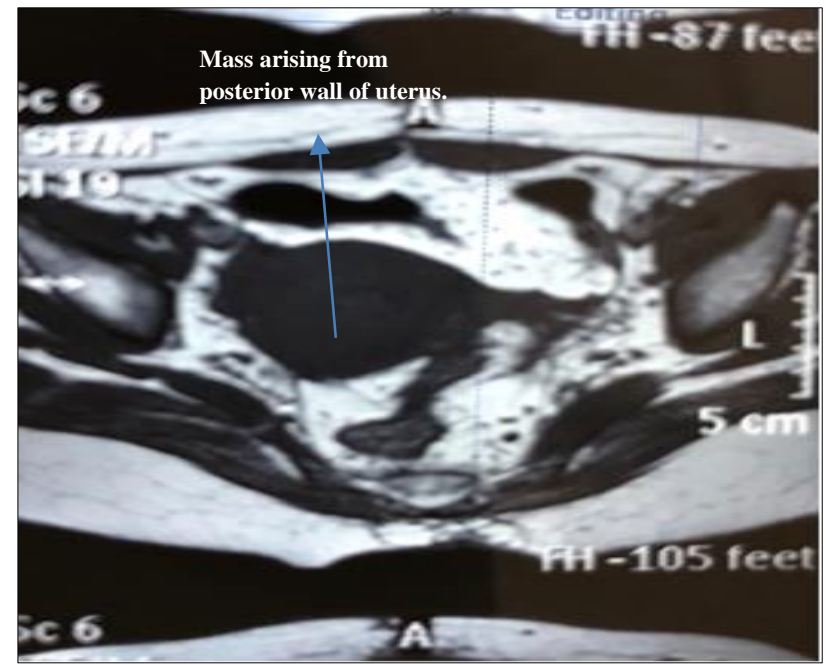

Figure 2: MRI pelvis of a large posterior wall fibroid with cystic degeneration.

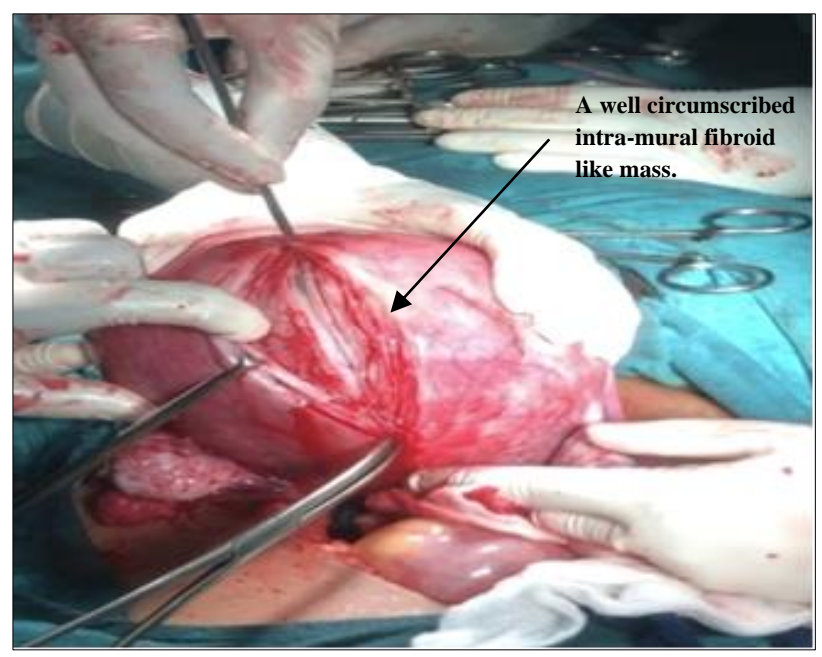

Figure 3: Intra operative finding of large posterior wall fibroid with cystic degeration.

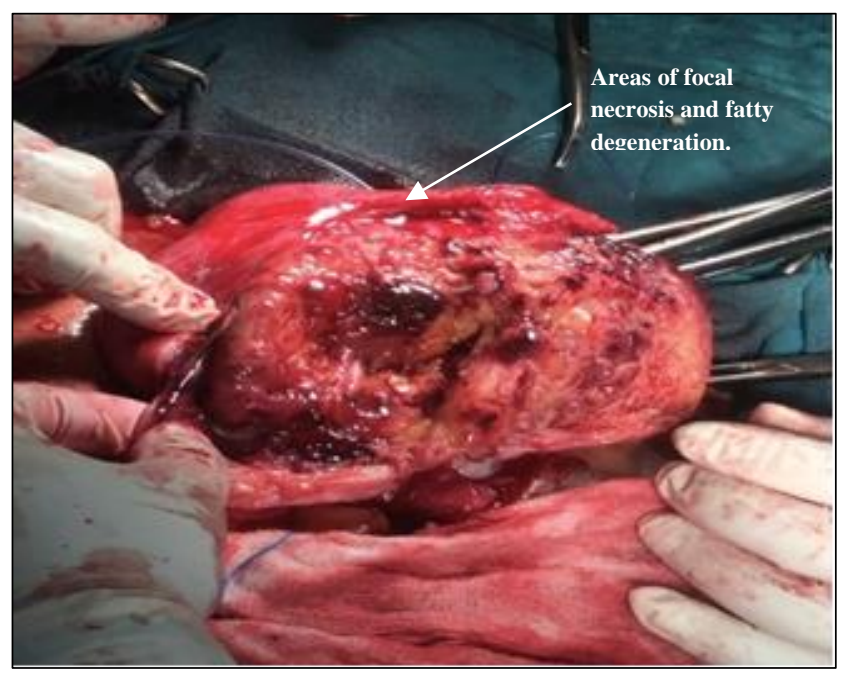

Figure 4: Gross appearance of the bed of lesion after draining the cystic fluid. 
Intraoperatively, a well circumscribed intramural fibroid like mass of size $8 \times 6 \mathrm{cms}$, with cystic degeneration containing $400 \mathrm{cc}$ serous fluid was resected successfully from posterior myometrium. It was challenging as cystic nature of the mass prevented us from using vasopressin to decrease blood loss, however bilateral uterine were occluded temporarily with a feeding tube, placed after creating a window in the broad ligament. Bilateral ovaries and tubes were healthy (Figure 3, 4). Histopathological evaluation of the specimen showed a well circumscribed tumour composed cells that closely resembled proliferative endometrial stromal cells which was surrounded by normal myometrial smooth muscles, minimal myometrial invasion i.e. $<3 \mathrm{~mm}$ and $<3$ in number with mitotic rate $<3 / 10 \mathrm{HPF}$, suggestive of endometrial stromal nodule (Figure 5, 6).

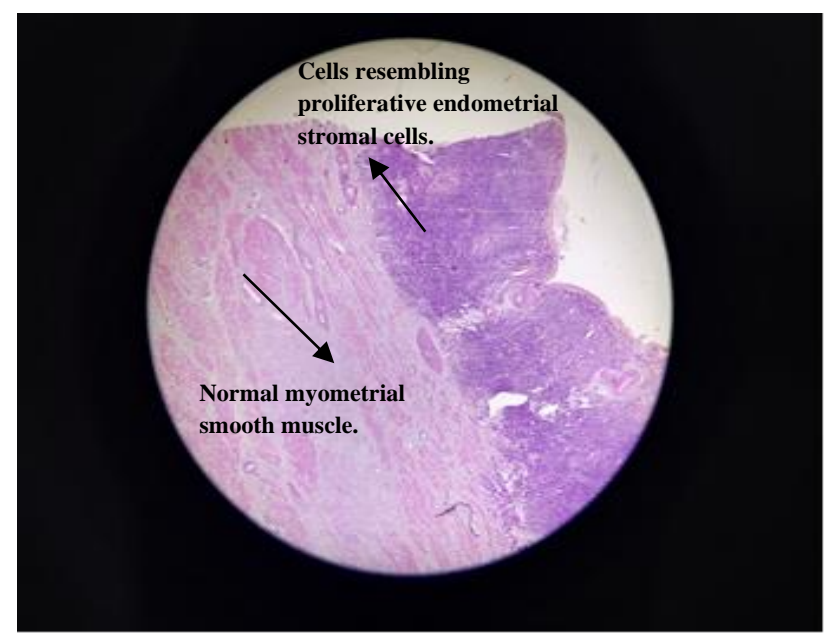

Figure 5: Histopathological view.



Figure 6: Microscopy ESN with a clear tumour myometrial interface.

\section{DISCUSSION}

Endometrial stromal tumours are among the least common neoplasm of uterus with an annual incidence of 2 per million women. ${ }^{4}$ Stromal nodules account for $1 / 4^{\text {th }}$ of the endometrial stromal tumours and are rare and benign subtype of stromal tumours. The clinical presentations are non-specific with the most common complain being abnormal uterine bleeding with or without pain abdomen and varying degrees of anemia. Sometimes diagnosed as accidental finding in hysterectomy specimen.

There is no definitive pre diagnostic test to diagnose the tumour, adequate sampling of the tumour-myometrial interface is necessary to evaluate the degree of infiltration of tumour into the myometrium and correctly classify the tumour. Therefore, despite being a benign entity hysterectomy is the gold standard. However, in a young patient who wants to retain fertility, as in our case, treatment can be individualized and conservative surgery or hormonal therapy can be tried.

The largest clinicopathological study so far on endometrial stromal nodules was conducted by Tavassoli FA and Norris HJ who studied 60 cases to determine their characteristics and behaviours. A follow up up to 16 years in these patients showed no sign of recurrences indicating its benign nature. ${ }^{2}$ Dionigi published a study of 50 cases of endometrial stromal tumours with limited or no infiltration at the margins out of which only 4 turned out to be endometrial stromal nodules, suggesting the rarity of ESNs. ${ }^{5}$ Most case reports in literature has reported ESN in the perimenopausal age group. ${ }^{6}$ Shilder et al published a case report with successful conservative management with leuprolide acetate, to preserve the fertility of a young nulliparous woman diagnosed with stromal nodule. ${ }^{7}$ Since this study patient is a nulliparous married women who is eager to conceive, and considering the margins of the stromal tumour was non infiltrative, with minimal mitotic activity and no lymphovascular invasion she was managed conservatively. The post-operative diagnosis was explained to the patient, regarding the benign nature of the tumour and need for follow up in upcoming years.

\section{CONCLUSION}

The rarity of these tumours and occurrence in perimenopausal age limits the clinical implementation of fertility preserving treatments such as local excision and/or endocrine therapy, endometrial stromal nodule in a young nulliparous female who underwent successful conservative surgery is a worth reporting.

\section{Funding: No funding sources Conflict of interest: None declared Ethical approval: Not required}

\section{REFERENCES}

1. Angelo ED, Prat J. Uterine sarcomas: a review. Gynecol Oncol. 2010;116(1)131-9.

2. Tavassoli FA, Norris HJ. Mesenchymal tumors of the uterus. VII. A clinicopathological study of 60 
endometrial stromal nodules. Histopathol. 1981;5(1):1-10.

3. Kim KR, Jun SY, Park IA, Ro JY, Nam JH. Endometrial stromal tumor with limited infiltration and probable extrauterine metastasis: report of a case. Ann Diagnost Pathol. 2005;9(1):57-60.

4. Zaloudek C, Hendrickson MR. Mesenchymal tumors of the uterus, in Blaustein's Pathology of the Female Genital Tract, New York. USA. 2001;5:561-615.

5. Dionigi A, Oliva E, Clement PB, Young RH. Endometrial stromal nodules and endometrial stromal tumors with limited infiltration: a clinicopathologic study of 50 cases. Am J Surg Pathol. 2002;26(5)567-81.

6. FdiliAlaoui FZ, Chaara H, Bouguern H, Melhouf MA, Fatemi H, Belmlih A, et al. Endometrial stromal nodule: report of a case. Case Reports Med. 2011;Article ID 260647.

7. Schilder JM, Hurd WW, Roth LM, Sutton GP. Hormonal treatment of an endometrial stromal nodule followed by local excision. Obstet Gynecol. 1999;93(5)(2)805-7.

Cite this article as: Bhattarai $\mathrm{S}$, Gupta $\mathrm{T}$, Bhardwaj J, Sablok A. Endometrial stromal nodule: a rare case report. Int J Reprod Contracept Obstet Gynecol 2020;9:2170-3. 